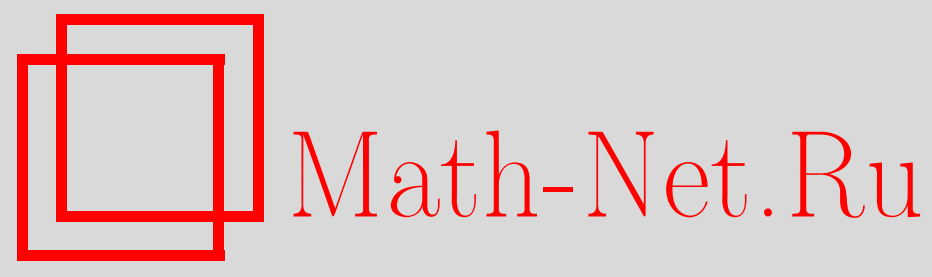

М. И. Тихомирова, Предельные распределения числа непоявившихся цепочек одинаковых исходов, Дискрет. матем., 2008, том 20, выпуск 3, 40-46

DOI: https://doi.org/10.4213/dm1011

Использование Общероссийского математического портала Math-Net.Ru подразумевает, что вы прочитали и согласны с пользовательским соглашением http://www . mathnet.ru/rus/agreement

Параметры загрузки:

IP : 44.207 .124 .84

26 апреля 2023 г., 03:16:55 


\title{
Предельные распределения числа непоявившихся цепочек одинаковых исходов
}

() 2008 г. М. И. Тихомирова

\begin{abstract}
Доказана асимптотическая нормальность числа непоявившихся $s$-цепочек одинаковых исходов в равновероятной полиномиальной схеме при условии, что число испытаний $n$ и число исходов $N$ стремятся к бесконечности так, что $\alpha_{N}=n / N^{s} \rightarrow \alpha$, $0 \leqslant \alpha<\infty, N \alpha_{N}^{2} \rightarrow \infty$.
\end{abstract}

\section{1. Введение}

Для выявления зависимостей, проявляющихся на длинных отрезках исследуемой последовательности

$$
x_{1}, x_{2}, \ldots, x_{t}, \ldots, \quad x_{t} \in \mathcal{N}=\{1,2, \ldots, N\},
$$

вместо статистик, зависящих от частот $s$-цепочек всего множества возможных значений $s$-цепочек, из-за сложности обработки реализаций последовательности (1) при больших $s$ используются только $s$-цепочки из некоторого подмножества $\mathscr{B}$ множества всех значений $s$-цепочек. В качестве подмножества $\mathscr{B}$ во многих работах (см., например, [1-5]) выбираются подмножества вида

$$
\mathscr{B}=\left\{\left(i_{1}, i_{2}, \ldots, i_{S}\right): i_{1}=i_{2}=\ldots=i_{s}, 1 \leqslant i_{1} \leqslant N\right\} .
$$

В работах $[1,2]$ исследовались предельные распределения статистик типа $\chi^{2}$, построенных по частотам $s$-цепочек множества (2). В работе [3] изучались статистики, связанные со сложными рекуррентными событиями, которые определялись моментами появления цепочек из множества $\mathscr{B}$. Статистики, равные числу непоявившихся $s$-цепочек из множества $\mathscr{B}$, изучались в [4].

Статистики типа числа пустых ящиков (см. $[4,6])$ можно представить в виде суммы зависимых величин. Методы исследования сходимости распределений сумм зависимых величин, предложенные С. Н. Бернштейном (см. [7]), были успешно применены для различных типов зависимостей, которые удобно описывать, используя разности номеров случайных величин, входящих в исследуемую сумму. Другие виды зависимостей оказалось удобнее описывать при помощи специально подобранных графов (см., например, $[8,9])$. В работе [4] для доказательства асимптотической нормальности числа непоявившихся $s$-цепочек использовались результаты работы [9]. Для числа $\mu_{0}(\mathscr{B})$ непоявившихся $s$-цепочек из множества $\mathscr{B}$ в [4] были получены только асимптотические формулы для $\mathbf{E} \mu_{0}(\mathscr{B})$ и $\mathbf{D} \mu_{0}(\mathscr{B}) ;$ условия теорем работы [9] в этом случае не выполняются. 
В данной работе будем предполагать, что случайные величины последовательности (1) независимы и равномерно распределены на множестве $\mathcal{N}$. Для доказательства асимптотической нормальности $\mu_{0}(\mathscr{B})$ используется подход, обобщающий один из трех подходов Реньи, примененных им к доказательству асимптотической нормальности числа пустых ящиков (см. [10, 6]).

\section{2. Производящие функции}

Обозначим через $\tau_{0}$ время до появления первой $s$-цепочки из множества $\mathscr{B}$, а через $\tau_{l}$ время, прошедшее между появлением $l$-й и $(l+1)$-й $s$-цепочек, $1 \leqslant l \leqslant N-1$. Эти величины, очевидно, независимы.

Случайная величина

$$
v_{k}(N)=\tau_{0}+\tau_{1}+\ldots+\tau_{k-1}
$$

есть время, прошедшее до первого появления $k$-й $s$-цепочки из множества $\mathscr{B}$.

Производящая функция $g_{0}(z)$ величины $\tau_{0}$ получена в работе [3]:

$$
g_{0}(z)=\frac{P_{0}(z)}{Q_{0}(z)}, \quad P_{0}(z)=N z^{s}-z^{s+1}, \quad Q_{0}(z)=(N-1) z^{s}-N^{s}(z-1) .
$$

Теорема 1. Производящую функцию $g_{l}(z)=\mathbf{E} z^{\tau_{l}}$ можно представить в виде

$$
g_{l}(z)=\frac{P_{l}(z)}{Q_{l}(z)}, \quad 0 \leqslant l \leqslant N-1,
$$

где

$$
\begin{aligned}
P_{l}(z) & =(N-l)\left(N z^{s}-z^{s+1}\right), \\
Q_{l}(z) & =z^{s+1} l+z^{s} N(N-l-1)+N^{s+1}(1-z) .
\end{aligned}
$$

Доказательство. Пусть $\mathscr{B}_{l}-$ некоторое подмножество множества $\mathscr{B}$ и $\left|\mathscr{B}_{l}\right|=N-l$, $\mathscr{B}_{0}=\mathscr{B}$. Определим цепь Маркова $\xi_{u}^{(l)}$ следующим образом: $\xi_{u}^{(l)}=k, 0 \leqslant k \leqslant s$, если $x_{u}=x_{u-1}=\ldots=x_{u-k+1} \neq x_{u-k}, \quad x_{u} \in \mathscr{B}_{0} \backslash \mathscr{B}_{l}$. Состояние $s$ будем считать поглощающим. Нулевым моментом времени считаем момент появления предыдущей $s$-цепочки, следовательно, $\xi_{0}^{(l)}=0$. Вероятности переходов $p_{i, j}^{(l)}=\mathbf{P}\left\{\xi_{t+1}^{(l)}=j \mid \xi_{t}^{(l)}=i\right\}$ этой цепи Маркова определяются равенствами

$$
\begin{array}{rlrl}
p_{k, k+1}^{(l)} & =\frac{1}{N}, & p_{k, 0} & =\frac{l}{N}, \\
p_{0,1}^{(l)} & =1-\frac{l}{N}, \quad p_{k, 1}^{(l)}=1-\frac{l+1}{N}, \quad 1 \leqslant k \leqslant s-1, \\
& =\frac{l}{N}, \quad p_{s, s}^{(l)}=1 .
\end{array}
$$

Время до попадания в поглощающее состояние совпадает с $\tau_{l}$. Введем обозначение

$$
g_{k}(l, z)=\mathbf{E}\left(z^{\tau_{l}} \mid \xi_{0}^{(l)}=k\right) .
$$

По формуле полного математического ожидания

$$
\begin{aligned}
\mathbf{E}\left(z^{\tau_{l}} \mid \xi_{0}^{(l)}=k\right)= & \frac{1}{N} \mathbf{E}\left(z^{\tau_{l}} \mid \xi_{0}=k, \xi_{1}=k+1\right) \\
& +\frac{l}{N} \mathbf{E}\left(z^{\tau_{l}} \mid \xi_{0}=k, \xi_{1}=0\right)+\left(1-\frac{l+1}{N}\right) \mathbf{E}\left(z^{\tau_{l}} \mid \xi_{0}=k, \xi_{1}=1\right) .
\end{aligned}
$$


Отсюда

$$
\begin{array}{rlrl}
g_{k}(l, z) & =z\left(\frac{1}{N} g_{k+1}(l, z)+\frac{l}{N} g_{0}(l, z)+\left(1+\frac{l+1}{N}\right) g_{1}(l, z)\right), & 1 \leqslant k \leqslant s-1, \\
g_{0}(l, z)=z\left(\left(1-\frac{l}{N}\right) g_{1}(l, z)+\frac{l}{N} g_{0}(l, z)\right), & g_{s}(l, z)=1 .
\end{array}
$$

Вычитая из первого равенства это же равенство с заменой $k$ на $k-1$, находим, что

$$
\begin{aligned}
g_{k+1}(l, z)-g_{k}(l, z) & =\frac{N}{z}\left(g_{k}(l, z)-g_{k-1}(l, z)\right), \quad 1 \leqslant k \leqslant s-1, \\
g_{1}(l, z)-g_{0}(l, z) & =\frac{N}{z} \frac{1-z}{N-l} g_{0}(l, z) .
\end{aligned}
$$

Отсюда нетрудно получить, что

$$
g_{0}(l, z)=\left(1+\frac{1-z}{N-l} \frac{N}{z}\left(1+\frac{N}{z}+\ldots+\left(\frac{N}{z}\right)^{s-1}\right)\right)^{-1} .
$$

Из этого равенства следует утверждение теоремы.

\section{3. Моменты времени до появления заданного числа цепочек}

Найдем сначала моменты величин $\tau_{l}$, определяющих величину $v_{k}(N)$ (см. (3)). Для моментов величин $\tau_{l}$ введем обозначения

$$
M_{k}^{(l)}=\mathbf{E} \tau_{l}^{k}, \quad \widehat{M}_{k}^{(l)}=\mathbf{E} \tau_{l}^{[k]},
$$

где $a^{[k]}=a(a-1) \ldots(a-k+1)$. Известно, что

$$
\widehat{M}_{k}^{(l)}=\left.\frac{d^{k} g_{l}(z)}{d z^{k}}\right|_{z=1}, \quad k=1,2, \ldots
$$

Дифференцируя четыре раза равенство $Q_{l}(z) g_{l}(z)=P_{l}(z)$ в точке $z=1$, получаем равенства

$$
\begin{aligned}
Q_{l}^{(1)}+Q_{l}^{(0)} g_{l}^{(1)} & =P_{l}^{(1)}, \\
Q_{l}^{(2)}+2 Q_{l}^{(1)} g_{l}^{(1)}+Q_{l}^{(0)} g_{l}^{(2)} & =P_{l}^{(2)}, \\
Q_{l}^{(3)}+3 Q_{l}^{(2)} g_{l}^{(1)}+3 Q_{l}^{(1)} g_{l}^{(2)}+Q_{l}^{(0)} g_{l}^{(3)} & =P_{l}^{(3)}, \\
Q_{l}^{(4)}+4 Q_{l}^{(3)} g_{l}^{(1)}+6 Q_{l}^{(2)} g_{l}^{(2)}+4 Q_{l}^{(1)} g_{l}^{(3)}+Q_{l}^{(0)} g_{l}^{(4)} & =P_{l}^{(4)},
\end{aligned}
$$

где

$$
\begin{aligned}
& P_{l}^{(0)}=Q_{l}^{(0)}=(N-l)(N-1), \\
& P_{l}^{(m)}=(N-l)\left(s^{[m]} N-(s+1)^{[m]}\right), \quad m \geqslant 1, \\
& Q_{l}^{(1)}=s((N-l)(N-1)-l)-N^{s+1}+l(s+1), \\
& Q_{l}^{(m)}=l(s+1)^{[m]}+((N-l)(N-1)-l) s^{[m]}, \quad 2 \leqslant m \leqslant s .
\end{aligned}
$$


Используя эти формулы, находим выражение для первого момента

$$
\mathbf{E} \tau_{l}=\widehat{M}_{l}^{(1)}=\frac{N}{N-l} \frac{N^{s}-1}{N-1}, \quad 0 \leqslant l \leqslant N-1 .
$$

Для моментов более высоких порядков получаются более сложные выражения. Приведем асимптотические формулы для моментов нужного нам порядка.

Лемма 1. При $N \rightarrow \infty$ равномерно по $l, 0 \leqslant l \leqslant N-1$, u $s \geqslant 2$

$$
\begin{gathered}
\widehat{M}_{m}^{(l)}=\frac{a_{m} N^{m s}}{(N-l)^{m}}\left(1+O\left(\frac{1}{N}\right)\right), \quad m=1, \ldots, 4, \\
a_{1}=1, \quad a_{2}=2, \quad a_{3}=6, \quad a_{4}=24 .
\end{gathered}
$$

Утверждение этой леммы нетрудно проверить, если воспользоваться равенствами (4) и (5).

Используя эту лемму и выражения центральных моментов $\tau_{l}$ через факториальные моменты, получим следующее утверждение.

Лемма 2. При $N \rightarrow \infty$ равномерно по $l, 0 \leqslant l \leqslant N-1$, u $s \geqslant 2$

$$
\mathbf{D} \tau_{l}=\frac{N^{2 s}}{(N-l)^{2}}\left(1+O\left(\frac{s}{N^{s-1}}\right)\right), \quad \mathbf{E}\left(\tau_{l}-\mathbf{E} \tau_{l}\right)^{4}=O\left(\frac{N^{4 s}}{(N-l)^{4}}\right) .
$$

Полученные выражения для моментов величины $\tau_{l}$ позволяют получить моменты и предельное распределение величины $v_{k}(N)$, определенной равенством (3).

\section{4. Моменты и предельное распределение величины $v_{k}(N)$}

Из определения (3) случайной величины $v_{k}(N)$ и равенства (6) следует, что

$$
\mathbf{E} v_{k}(N)=N \frac{N^{s}-1}{N-1} \sum_{l=N-k+1}^{N} \frac{1}{l} \text {. }
$$

Теорема 2. Если при $N \rightarrow \infty$ параметры $k$ u s меняются так, что

$$
\gamma_{N}=k / N \rightarrow \gamma, \quad 0 \leqslant \gamma<1, \quad k^{2} / N \rightarrow \infty,
$$

то равномерно по $s \geqslant 2$

$$
\mathbf{E} v_{k}(N)=N^{s} \ln \frac{1}{1-\gamma_{N}}+O\left(N^{s-1}\right), \quad \mathbf{D} v_{k}(N)=N^{2 s-1} \frac{\gamma_{N}}{1-\gamma_{N}}(1+O(1 / k)) .
$$

Доказательство. Первое равенство следует из равенства (6) и неравенства

$$
\ln N-\ln (N-k+1) \leqslant \sum_{l=N-k+1}^{N} \frac{1}{l} \leqslant \ln (N-1)-\ln (N-k),
$$

получающегося интегрированием неравенства $x^{-1} \leqslant[x]^{-1} \leqslant(x-1)^{-1}$ в пределах от $N-k+1$ до $N+1$. Второе равенство получается из равенства

$$
\mathbf{D} v_{k}(N)=\sum_{l=0}^{k-1} \mathbf{D} \tau_{l}
$$


леммы 2 и неравенства

$$
\frac{k-1}{(N+1)(N-k+1)} \leqslant \sum_{l=N-k+1}^{N} \frac{1}{l^{2}} \leqslant \frac{k}{N(N-k)},
$$

которое доказывается интегрированием неравенства $x^{-2} \leqslant[x]^{-2} \leqslant(x-1)^{-2}$ в пределах от $N-k+1$ до $N+1$.

Теорема 3. Если при $N \rightarrow \infty$ параметры $k$ u $s, s \geqslant 2$, меняются так, что

$$
\gamma_{N}=k / N \rightarrow \gamma, \quad 0 \leqslant \gamma<1, \quad k^{2} / N \rightarrow \infty,
$$

то равномерно по $x,-\infty<x<\infty$,

$$
\lim _{N \rightarrow \infty} \mathbf{P}\left\{\frac{v_{k}(N)+N^{s} \ln \left(1-\gamma_{N}\right)}{\sqrt{N^{2 s-1} \gamma_{N} /\left(1-\gamma_{N}\right)}}<x\right\}=\Phi(x),
$$

где

$$
\Phi(x)=\frac{1}{\sqrt{2 \pi}} \int_{-\infty}^{x} \exp \left\{\frac{-u^{2}}{2}\right\} d u .
$$

Доказательство. Условие Ляпунова для суммы (3) можно записать в виде

$$
L(k, N)=\frac{\sum_{l=0}^{k-1} \mathbf{E}\left(\tau_{l}-\mathbf{E} \tau_{l}\right)^{4}}{\left(\mathbf{D} v_{k}(N)\right)^{2}} \rightarrow 0
$$

при $N \rightarrow \infty$. Из леммы 2 следует, что

$$
\sum_{l=0}^{k-1} \mathbf{E}\left(\tau_{l}-\mathbf{E} \tau_{l}\right)^{4} \leqslant C N^{4 s} \sum_{l=0}^{k-1} \frac{1}{(N-l)^{4}} .
$$

В условиях теоремы

$$
\sum_{l=0}^{k-1} \frac{1}{(N-l)^{4}}=\sum_{l=N-k+1}^{N} \frac{1}{l^{2}} \leqslant C\left(\frac{1}{(N-k+1)^{3}}-\frac{1}{N^{3}}\right)=C \frac{1}{N^{3}}\left(\frac{1}{1-\gamma_{N}}-1\right) .
$$

Отсюда, из (9) и неравенства

$$
\left(\mathbf{D} v_{k}(N)\right)^{2}>c_{0} N^{4 s-2}\left(\frac{\gamma_{N}}{1-\gamma_{N}}\right)^{2}, \quad c_{0}>0,
$$

которое следует из теоремы 2, получаем, что

$$
L(k, N)<C \frac{N^{4 s-3}}{N^{4 s-2}}=C / N \rightarrow 0
$$

при $\gamma_{N} \rightarrow \gamma>0$.

Если $\gamma=0$, то

$$
\left(\mathbf{D} v_{k}(N)\right)^{2} \geqslant c_{0} N^{4 s-2}\left(\frac{k}{N}\right)^{2}
$$

и при $N \rightarrow \infty$

$$
L(k, N)<C / k \rightarrow 0 .
$$

Таким образом, условие (8) выполнено. Теорема доказана. 


\section{5. Предельная теорема для числа непоявившихся цепочек}

Число $\mu_{0}(n, N)$ непоявившихся $s$-цепочек из множества $\mathscr{B}$ в $n+s-1$ первых членах последовательности (1) можно представить в виде

$$
\mu_{0}(n, N)=\sum_{i=1}^{N} \chi\left(\left(x_{t}, \ldots, x_{t+s-1}\right) \neq(i, \ldots, i), t=1, \ldots, n\right) .
$$

Если $n, N \rightarrow \infty$ так, что $n / N^{s}=\alpha_{N} \rightarrow \alpha, 0<\alpha<\infty$, то (см. $\left.[4,5]\right)$ при любом $\delta>0$

$$
\begin{aligned}
& \mathbf{E} \mu_{0}(n, N)=N \exp \left\{-\alpha_{N}\right\}+\alpha_{N} \exp \left\{-\alpha_{N}\right\}+O\left(\frac{1}{N^{1-\delta}}\right), \\
& \mathbf{D} \mu_{0}(n, N)=N \exp \left\{-\alpha_{N}\right\}\left(1-\exp \left\{-\alpha_{N}\right\}\right)+O\left(N^{\delta}\right) .
\end{aligned}
$$

Теорема 4. Если $n, N, s, s \geqslant 2$, при $N \rightarrow \infty$ изменяются так, что

$$
\alpha_{N}=n / N^{s} \rightarrow \alpha, \quad 0 \leqslant \alpha<\infty, \quad N \alpha_{N}^{2} \rightarrow \infty,
$$

mo

$$
\lim _{N \rightarrow \infty} \mathbf{P}\left\{\frac{\mu_{0}(n, N)-N \exp \left\{-\alpha_{N}\right\}}{\sqrt{N \exp \left\{-\alpha_{N}\right\}\left(1-\exp \left\{-\alpha_{N}\right\}\right)}}<x\right\}=\Phi(x) .
$$

Доказательство. Воспользуемся теоремой 3 и равенством

$$
\mathbf{P}\left\{v_{k}(N) \leqslant n\right\}=\mathbf{P}\left\{\mu_{0}(n, N) \leqslant N-k\right\} .
$$

Выберем $k$ так, чтобы при $N \rightarrow \infty$ выполнялось равенство

$$
N-k-N \exp \left\{-\alpha_{N}\right\}=x_{N} \sqrt{N \exp \left\{-\alpha_{N}\right\}\left(1-\exp \left\{-\alpha_{N}\right\}\right)},
$$

где $x_{N} \rightarrow x$. Отсюда

$$
k=N\left(1-z_{N}\right)-x_{N} \sqrt{N z_{N}\left(1-z_{N}\right)}, \quad z_{N}=\exp \left\{-\alpha_{N}\right\}, \quad 0<z_{N}<1 .
$$

Следовательно,

$$
z_{N}=F\left(z_{N}\right)
$$

где

$$
F(z)=1-\gamma_{N}-\frac{x_{N}}{N} \sqrt{z(1-z)}, \quad \gamma_{N}=k / N,
$$

и при $N \rightarrow \infty$

$$
\gamma_{N}=\frac{k}{N} \rightarrow \gamma=1-\exp \{-\alpha\} .
$$

Из равенства $z_{N}=F\left(F\left(z_{N}\right)\right)$ получаем, что

$$
z_{N}=1-\gamma_{N}-\frac{x_{N}}{\sqrt{N}} \sqrt{\left(1-\gamma_{N}-\frac{x_{N}}{\sqrt{N}} \sqrt{z_{N}\left(1-z_{N}\right)}\right)\left(\gamma_{N}+\frac{x_{N}}{\sqrt{N}} \sqrt{z_{N}\left(1-z_{N}\right)}\right)} .
$$


Отсюда

$$
\begin{aligned}
& z_{N}=1-\gamma_{N}-\frac{x_{N}}{\sqrt{N}} \sqrt{\gamma_{N}\left(1-\gamma_{N}\right)}+O\left(\frac{1}{N}\right), \\
& \alpha_{N}=-\ln \left(1-\gamma_{N}\right)+\frac{x_{N}}{\sqrt{N}} \sqrt{\frac{\gamma_{N}}{1-\gamma_{N}}}+O\left(\frac{1}{N}\right) .
\end{aligned}
$$

Таким образом,

$$
n=-N^{s}\left(\ln \left(1-\gamma_{N}\right)-x_{N} \frac{1}{\sqrt{N}} \sqrt{\frac{\gamma_{N}}{1-\gamma_{N}}}+O\left(\frac{1}{N}\right)\right) .
$$

Подставляя это выражение для $n$ в левую часть равенства (10), по теореме 3 получаем, что при $N \rightarrow \infty$

$$
\mathbf{P}\left\{v_{k}(N) \leqslant n\right\}=\mathbf{P}\left\{\frac{v_{k}(N)+N^{s} \ln \left(1-\gamma_{N}\right)}{N^{s} \sqrt{\gamma_{N} /\left(\left(1-\gamma_{N}\right) \sqrt{N}\right)}}<x_{N}+O\left(\frac{1}{N}\right)\right\} \rightarrow \Phi(x),
$$

так как выполнены условия (14). Отсюда и из (10) и (11) следует утверждение теоремы.

\section{Список литературы}

1. Башарин Г. П., Об использовании критерия согласия $\chi^{2}$ в качестве критерия независимых испытаний. Теория вероятностей и ее применения (1957) 2, №1, 141-142.

2. Тихомирова М. И., Предельные распределения статистик $\chi^{2}$ для частот цепочек одинаковых исходов полиномиальной схемы. Труды по дискретной математике (2003) 7, 191-200.

3. Тихомирова М. И., Чистяков В. П., Предельные распределения некоторых статистик, связанных с рекуррентными событиями. Труды по дискретной математике (2003) 7, 201-212.

4. Тихомирова М. И., Предельные теоремы для числа непоявившихся s-цепочек, Диссертация на соискание ученой степени кандидата физико-математических наук. МИЭМ, Москва, $1998 .$.

5. Тихомирова М. И., Чистяков В. П., Об асимптотике моментов числа непоявившихся $s$-цепочек. Дискретная математика (1997) 9, №1, 12-29.

6. Колчин В. Ф., Севастьянов Б. А., Чистяков В. П., Случайные размещения. Наука, Москва, 1976.

7. Бернштейн С. Н., Собрание сочинений, 6. Наука, Москва, 1964.

8. Janson S., Normal convergence by higher semiinvariants with application to sums of dependent random variables and random graphs. Ann. Probab. (1988) 16, №1, 305-312.

9. Михайлов В. Г., Асимптотическая нормальность разделимых статистик от частот $m$-цепочек. Дискретная математика (1989) 1, №4, 92-103.

Статья поступила 14.09.2006. Переработанный вариант поступил 2.03.2007. 